\title{
A Smart Shoe for Building a Real-Time 3D Map
}

\author{
Luan V. Nguyen ${ }^{1}$, Hung M. $\mathrm{La}^{1}$, Jesus Sanchez ${ }^{1}$ and $\mathrm{Tam} \mathrm{Vu}^{2}$ \\ Department of Computer Science and Engineering, University of Nevada at Reno, USA ${ }^{1}$ \\ Department of Computer Science and Engineering, University of Colorado at Denver, USA ${ }^{2}$
}

\begin{abstract}
Three dimensional (3D) mapping of environments has gained tremendous attention, from academic to industry to military, owing to the ever increasing needs of environmental modeling as well as monitoring. While many highly effective techniques have been reported thus far, a few even turned into commercial products, none has explored the use of wearable sensors to capture human foot motion, gait, and phase for 3D map construction, especially in the Architecture, Engineering, and Construction (AEC) domain. In this work, we propose a smart (and wearable) shoe, called "Smart Shoe", which integrates multiple laser scanners and an inertial measurement unit (IMU) to build a 3D map of environments in real time. Such a Smart Shoe can be a potential tool for floor plan surveying, in-construction monitoring, planning renovations, space usage planning, managing building maintenance and other tasks in the AEC domain. Besides, this Smart Shoe could assist disabled people (blind people) to navigate and avoid obstacles in the unknown environment. In another case, the shoes may help firefighters quickly model and recognize objects in the firing, dark, and smoky buildings where traditional camera-based approaches might not be applicable. We integrate this shoe with a novel foot localization algorithm that produces a smooth and accurate pose and trajectory of human walking, which is the key enabling technique to minimize data registration errors from laser point cloud.
\end{abstract}

Key words: Robotics, 3D Map, Real-time 3D Map Algorithms, SLAM.

\section{Introduction}

Three dimensional (3D) mapping of an unknown environment is open and important research since it has broad applications in environmental searching, automation in construction,

5 exploring and monitoring [1, 2]. Laser scanners can be used to collect 2D measurements of a facility's as-built condition, and the resulting point cloud can be processed to construct a 3D map for the Building Information Models (BIMs) [3]. There are successful research efforts in this field, and some of which have 10 been turned into commercial products such as Velodyne LiDar [4]. Integration of laser scanners or 3D LiDar sensor to build a 3D map of the environment is reported in [5, 6, 7, 8]. Their proposed 3D mapping systems integrated on a mobile vehicle can work in large scale environments, but they are not appro15 priate to a normally worm device such as the Smart Shoe in this paper. Due to natural localization challenges $[9,10,11,4]$ of $^{40}$ human foot motion based wearable sensors, not much research on 3D real-time mapping wearable sensor devices on the foot has been reported yet.

The original idea for this paper started from our observation of human foot motion gait of which we can exploit to design a Smart Shoe which is a compact wearable device. This Smart Shoe with 3D real-time mapping abilities has potential applications such as: helping visually impaired users improve their

This work was supported by the University of Nevada at Reno, and the National Science Foundation under grants: NSF-NRI\# 1426828 and NSF-IIP \#1559942.

*Corresponding author: Hung M. La; Email: hla@unr.edu quickly model and recognize objects in the fire and dark smoky buildings where cameras may not be useful.

In the Architecture, Engineering, and Construction (AEC) domain, the Smart Shoe can be used for floor plan surveying, in-construction monitoring, planning renovations, space usage planning, managing building maintenance. Since the Smart Shoe is lightweight and works in real-time it can be used as a construction safety monitoring and training tool.

This Smart Shoe implementation with wearable sensors including laser scanners and an inertial measurement unit (IMU) faced several challenges. First, it requires a very compact design for the natural movement of feet, such as: fast walking, climbing up or down, running or jumping. Second, the accuracy of tracking position and orientation of foot motion is crucial in building a 3D map [12, 13]. Finally, because the swing of laser scanners during foot motion definitely increases the noise of collected data, it obviously reduces the accuracy of 3D map result.

In order to deal with these challenges, we utilized the human foot motion gait to implement a human foot motion localization algorithm for accurate and smooth foot motion position, and to swing laser scanners how they could scan a full 3D mapping of environments. The results of this proposed Smart Shoe could be a frame work for other potential researches and applications of mobile wearable devices for 3D mapping in real-time. The initial report of this work has been presented in [14].

The remainder of the paper is organized as follows. The next section presents an overview of the Smart Shoe design, and data 
collection and processing. Section 3 presents a real-time human

${ }_{55}$ foot motion localization scheme. The 3D mapping algorithm for the Smart Shoe is presented in Section 4. Section 5 presents experimental results to demonstrate the effectiveness of the proposed Smart Shoe. Finally, an extended discussion of possible applications of the Smart Shoe in ACE and future work con60 cludes the paper in the conclusion section, Section 6 .

\section{Overall Design of a Smart Shoe}

In order to help reader be insightful the methodology of this Smart Shoe and how it could exploit gait phases of human foot motion to build a 3D map of environments, this section shows an overview of the proposed Smart Shoe design and how this design guarantees for successfully building a 3D map.

\subsection{Design of a Smart Shoe}

The Smart Shoe sketch and its components are depicted in Fig. 1 and Fig. 2, respectively. There is one IMU sensor 70 mounted on the front top of the shoe to help localize the foot during walking. Additionally, two 2D laser range scanners are mounted on the front and rear of the shoe to scan the surrounding environment. Data from these IMU and laser range scanners are transfered to laptop computer for real-time data processing.

75 Both real-time human foot localization and 3D mapping algorithms (Fig. 2) run in the computer to create a 3D map of the surrounding environment during the foot motion.

Mounting the laser range scanners on the shoe/foot has several advantages comparing to mounting on other body parts.

so First, by utilizing the foot motion during the walk, a 3D scanning field can be created without using other supporting actuation mechanisms such as motors to rotate the laser scanners, and this can help save energy/power. Likewise, mounting on the wearer's chest, back, or head leads to a need of an actua85 tor to rotate the laser scanner in order to have a 3D scanning field. Second, the foot motion has two different phases, stance and swing, as shown in Fig. 3 which can be utilized to reduce IMU drift to enhance the accuracy of the foot localization. The higher accuracy of foot localization, the better accuracy of the

90 3D map. Third, it is more convenient and easier to mount laser scanners on the shoe than on other body parts.

In this design (Fig. 1), the S martS hoe frame is considered as a rigid body frame [15] which includes three sub-body frames: Laser S canner 1 on the front of the shoe, IMU under the front

95 laser scanner, and Laser Scanner 2 on the rear of the shoe. The distance between the two centers, $O_{1}$ of the Laser Scanner $1^{115}$ frame and $\mathrm{O}_{2}$ of the Laser Scanner 2 frame, is $d_{\mathrm{O}_{1} \mathrm{O}_{2}}$, and an axis $O_{1} O_{2}$ is parallel to the ground. Because the body frame of the Laser Scanner 1 is setup to be the same as the Smart Shoe frame, $O_{1}$ is equivalent to $O_{S}$ of the Smart Shoe frame.

Likewise, the $I M U$ frame is also equivalent to the $S$ mart $S$ hoe frame, hence the original center $O_{I}$ of $I M U$ frame, 120 $O_{S}$ of Smart Shoe frame, and $O_{1}$ of Laser Scanner 1 frame are equivalent, or $O_{S} \equiv O_{1} \equiv O_{I}$, as shown in Fig. 1. This leads to the angle rate of IMU (Roll, Pitch, Yaw) during foot movement be also the angle rate of Laser Scanner 1 and Smart Shoe frames, respectively.

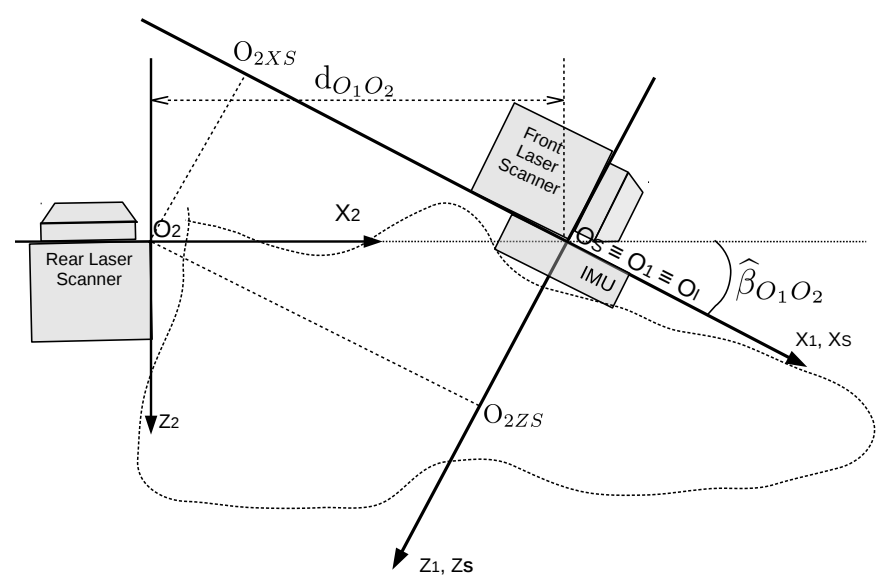

Figure 1: The Smart Shoe design.

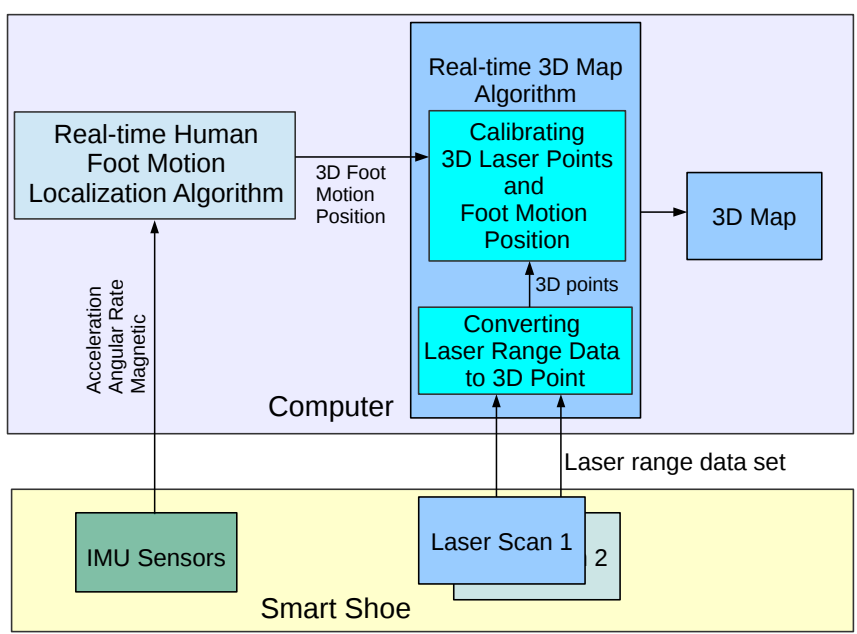

Figure 2: The Smart Shoe's Components.

Since two laser range scanners are mounted on different positions, they have different detected/scanned areas, detected distances, number of points per scanning, and scanning frequencies. In this design, because the coordinate systems of Smart Shoe and Laser Scanner 1 are setup equivalently to IMU coordinate system, their $\mathrm{Z}$ axis is pointing toward the ground as shown in Fig. 1. Also, $X_{1}, X_{I}$ and $X_{S}$ are equivalent to the Roll axis; $Y_{1}, Y_{I}$ and $Y_{S}$ are equivalent to the Pitch axis; and finally $Z_{1}, Z_{I}$ and $Z_{S}$ are equivalent to the $Y a w$ axis.

\subsection{Scanning Field of the Smart Shoe}

Because a crucial requirement for this Smart Shoe to successfully build a 3D map is its capability of helping laser scanners scan fully 3D sphere space above the ground, missing any portion of this space can lead to the failure of building a full $3 \mathrm{D}$ map of environments. When observing gait phases of human foot motion (see Fig. 3), we discovered that the changing angle of foot motion could help the front and rear laser scanners scan a maximum angle of $5 \pi / 6$ around the Pitch axis. This maximum 
angle, of course, depends on walking speeds [16] [17]. Hence, 130 by integrating two laser range scanners on the rear and front of a shoe, the scanning field can reach to a maximum covered angle $5 \pi / 6$ such as in Fig. 4 .
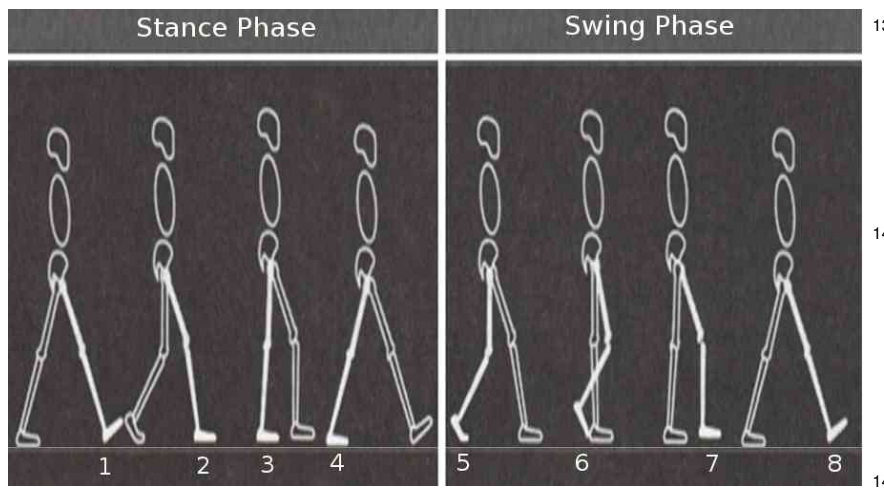

Figure 3: The Human Motion Gait Phase.

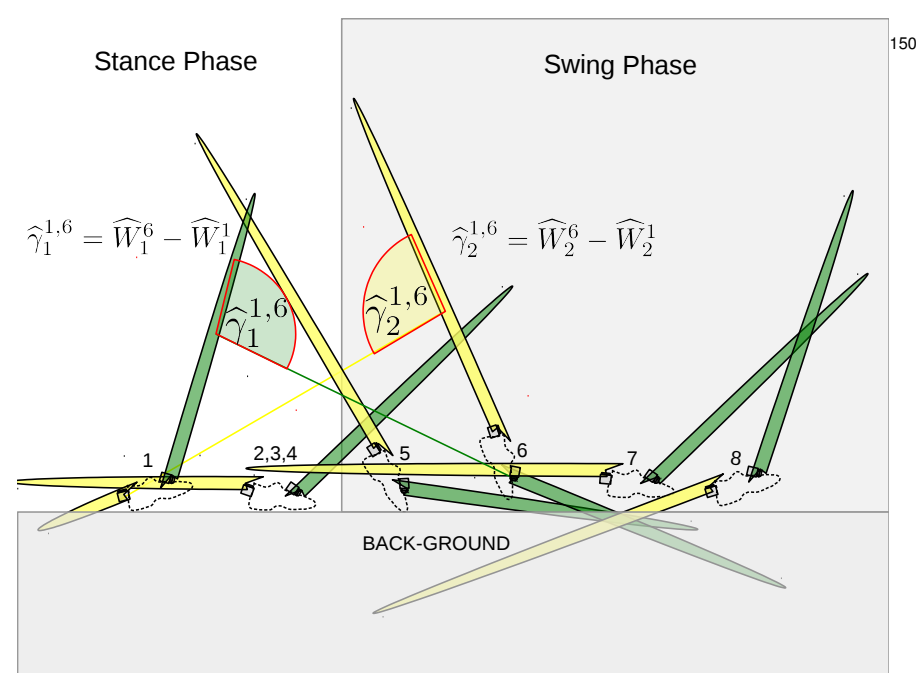

Figure 4: The Smart Shoe Scanning Field by Gait phase.

In Fig. 4 the yellow and green major sectors are the detected areas of the Laser Scanner 2 and the Laser Scanner 1, respectively. The 3D space volume covered by the yellow and green color areas is the scanning field of the Smart Shoe moving from the stance phase to the swing phase in one step. Furthermore, the gait and positions of this movement can be divided into two ${ }_{155}$ phases and 8 positions [16] [17] as shown in Fig. 3 . The maximum scanning angle of Laser Scanner 1, $\widehat{\gamma}_{1}^{1,6}$, and Laser Scanner $2, \widehat{\gamma}_{2}^{1,6}$, can be estimated at positions 1 and 6 of the human gait phase in Fig. 3 and Fig. 4 Therefore, a total physical scanning angle composed by both Laser Scanners 1 and Laser ${ }_{160}$ Scanner 2 is obtained as followed:

$$
\begin{gathered}
\widehat{\gamma}_{1 U}^{1,6}=\widehat{\gamma}_{1}^{1,6}-\widehat{\gamma}_{1 L}^{1,6} \\
\widehat{\gamma}_{2 U}^{1,6}=\widehat{\gamma}_{2}^{1,6}-\widehat{\gamma}_{2 L}^{1,6} \\
\widehat{\gamma}_{b}=\pi-\left(\widehat{\gamma}_{1 U}^{1,6}+\widehat{\gamma}_{2 U}^{1,6}\right),
\end{gathered}
$$

where $\widehat{\gamma}_{1 U}^{1,6}, \widehat{\gamma}_{2 U}^{1,6}$ are real scanning angles of Laser Scanner 1 and Laser Scanner 2 above the ground, respectively; $\widehat{\gamma}_{b}$ is unscanned angle of this Smart Shoe. The estimation value of the un-scanned angle is $\widehat{\gamma}_{b}\left(\pi / 6 \leq \widehat{\gamma}_{b} \leq \pi / 3\right)$ as shown in Fig. 5

Obviously, the un-scanned angle of the Smart Shoe depends on walking speeds too, and it may fluctuate from $\pi / 6$ to $\pi / 3$. At a normal walking speed, it is approximate to $\pi / 4$. However, this lacking volume can be compensated by the scanning volume of a next step. Therefore, this design can guarantee to fully scan a 3D map of the half sphere above the ground of environments during foot motion. This is the advantage of mounting laser range scanners on the foot.

\subsection{Data Collecting and Processing}

Based on the design of the Smart Shoe presented in the previous section, we can compute 3D positions of scanning points from laser scanners in their body frames and then transform them to the North East Down (NED) frame [18][19]. Without loss of generality, we assumed that the Laser Scanner 1 and the Laser Scanner 2 are different laser scanners. Hence, the detection areas $d A_{1}^{0}, d A_{2}^{0}$ are different as shown in Fig. 5 Also, the maximum detected distance at any scanning time $t$ of any point $i$ of Laser Scanner 1, $d_{1}^{t}(i)$ and that of Laser Scanner 2, $d_{2}^{t}(i)$ are different; and the number of points for each degree of Laser Scanner 1, $n P_{1}$ and Laser Scanner 2, $n P_{2}$ are also different.

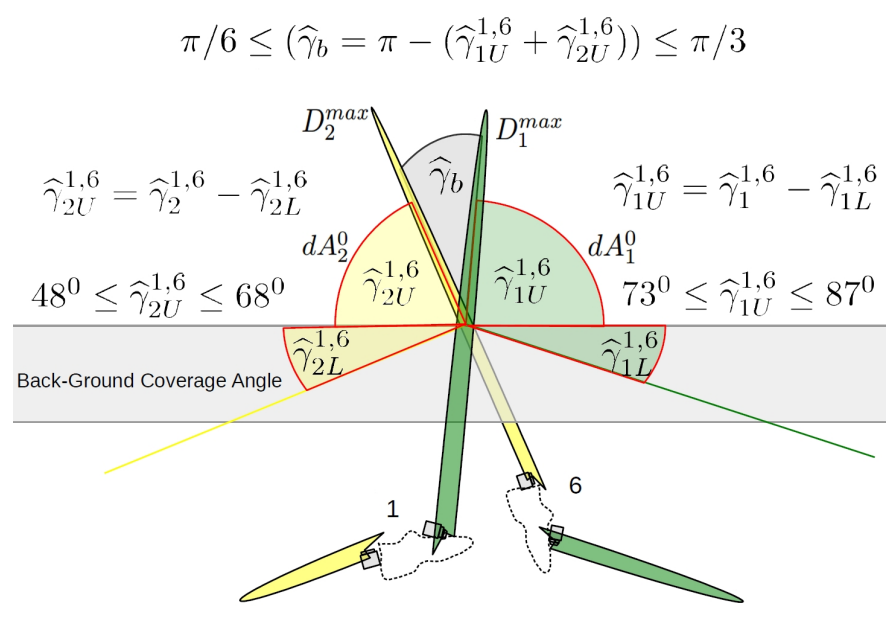

Figure 5: The Smart Shoe Scanning Field Estimation.

The Laser Scanner 1 and Laser Scanner 2 have total scanning points of scanning ranges $D_{1}^{\max } * n P_{1}$ and $D_{2}^{\max } * n P_{2}$, respectively. $D_{1}^{\max }$ and $D_{2}^{\max }$ are the maximum scanning range of laser scanner 1 and 2, respectively. For example, Hokuyo laser range scanner URG-04LX-UG01 has the maximum scanning range of $240^{\circ}$, but UST-10LX has the maximum scanning range of $270^{\circ}$. The duration for one full scanning of Laser Scanner 1 is $\delta_{1} t$ and Laser Scanner 2 are $\delta_{2} t, \delta_{1} t \neq \delta_{2} t$, respectively. The duration time for IMU sensor respondent is $\delta_{I} t$ which normally is thousand times smaller than $\delta_{1} t$ and $\delta_{2} t$.

In order to build a 3D map, we need to calculate scanning point of each laser scanner and transform them into the $N E D$ frame system as discussed in the following subsections. 


\subsubsection{Front Laser Scanner 1 Data}

In this subsection, we convert each point from Laser Scanner 1 into its 3D body frame such as in Fig. 6

The 3D coordinate values of any point $i$ at a discrete scanning ${ }_{185}$ time $t$ of Laser Scanner 1 is obtained as follows:

$$
\begin{gathered}
x_{1}^{t}(i)=0.0 \\
y_{1}^{t}(i)=d_{1}^{t}(i) * \cos \left(\alpha_{1}^{t}(i)\right) \\
z_{1}^{t}(i)=d_{1}^{t}(i) * \sin \left(\alpha_{1}^{t}(i)\right)
\end{gathered}
$$

Whereas $\alpha_{1}^{t}(i)$ and $i$

if $i_{1}^{\min } \leq i<i_{1}^{0}$ then $\alpha_{1}^{\min } \leq \alpha_{1}^{t}(i)<2 \pi$; if $i_{1}^{0} \leq i \leq i_{1}^{\max }$ then $0.0 \leq \alpha_{1}^{t}(i) \leq \alpha_{1}^{\max }$;

$i_{1}^{\min }=0, \quad i_{1}^{0}=\left(\left(D_{1}^{\max }-\pi\right) / 2-\pi\right) * n P_{1} ; \quad i_{1}^{\max }=D_{1}^{\max } * n P_{1} ;$ 175 $\alpha_{1}^{\min }=\left(\pi-\left(D_{1}^{\max }-\pi\right) / 2\right), \quad \alpha_{1}^{0}=0.0 ; \quad \alpha_{1}^{\max }=\left(D_{1}^{\max }-\pi\right) / 2$

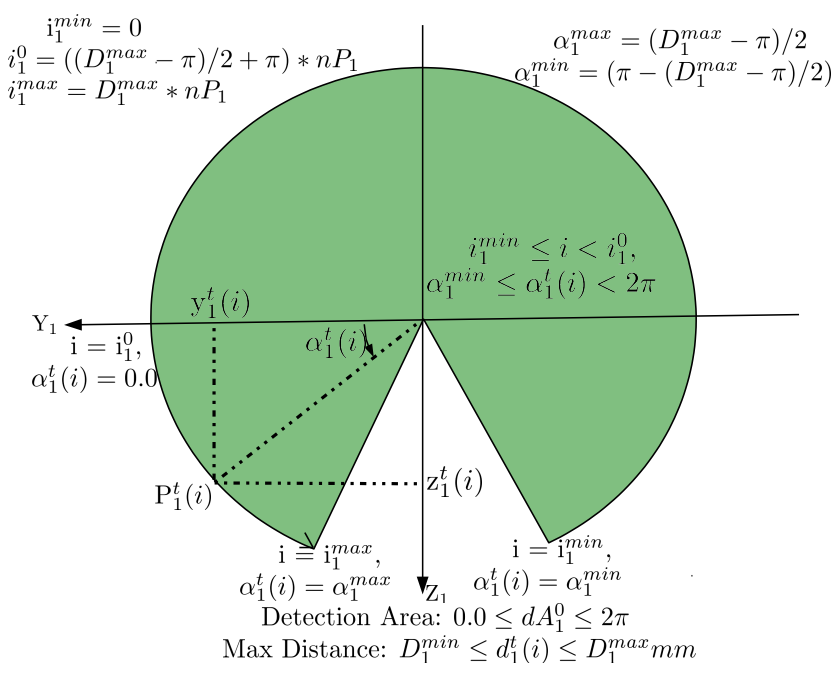

Figure 6: Field of View of the front Laser Scanner 1.

Because $O_{S} \equiv O_{1} \equiv O_{I}$, we can easily transform a Laser Scanner 1's 3D point coordinate $\left(x_{1}^{t}(i), y_{1}^{t}(i), z_{1}^{t}(i)\right)$ into the Smart Shoe frame $\left(x_{1 S S}^{t}(i), y_{1 S S}^{t}(i), z_{1 S S}^{t}(i)\right)$ as followed:

$$
\begin{aligned}
& x_{1 S S}^{t}(i)=x_{1}^{t}(i) \\
& y_{1 S S}^{t}(i)=y_{1}^{t}(i) \\
& z_{1 S S}^{t}(i)=z_{1}^{t}(i)
\end{aligned}
$$

The subscript ${ }_{S S}$ in these equations stands for the Smart Shoe ${ }_{190}$ body frame.

Last but not least important, we need to transform this 3D coordinate values from Smart Shoe frame into the NED frame as presented in subsection 2.4

\subsubsection{Rear Laser Scanner 2 Data}

The field of view of the Laser Scanner 2 is explained in Fig. 7. Equations to convert a distance of any point $i$ in any discrete scanning time $t$ from Laser Scanner 2 into a 3D coordinate are:

$$
\begin{gathered}
x_{2}^{t}(i)=d_{2}^{t}(i) * \sin \left(\alpha_{2}^{t}(i)\right) \\
y_{2}^{t}(i)=d_{2}^{t}(i) * \cos \left(\alpha_{2}^{t}(i)\right) \\
z_{2}^{t}(i)=0.0
\end{gathered}
$$

Whereas $\alpha_{2}^{t}(i)$ and $i$ :

if $\quad i_{2}^{\min } \leq i<i_{2}^{0} \quad$ then $\quad \alpha_{2}^{\min } \leq \alpha_{2}^{t}(i)<2 \pi ; \quad$ if $\quad i_{2}^{0} \leq i \leq$ $i_{2}^{\max }$ then $0.0 \leq \alpha_{2}^{t}(i) \leq \alpha_{2}^{\max }$;

$i_{2}^{\min }=0, \quad i_{2}^{0}=\left(\left(D_{2}^{\max }-\pi\right) / 2-\pi\right) * n P_{2} ; \quad i_{2}^{\max }=D_{2}^{\max } * n P_{2} ;$ $\alpha_{2}^{\min }=\left(\pi-\left(D_{2}^{\max }-\pi\right) / 2\right), \quad \alpha_{2}^{0}=0.0 ; \quad \alpha_{2}^{\max }=\left(D_{2}^{\max }-\pi\right) / 2$.

The rear Laser Scanner 2 frame has a slightly different $\widehat{\beta}_{O_{1} O_{2}}$ angle than the others, and it has a distance $d_{O_{1} O_{2}}$ from its body frame to the Laser Scanner 1 frame. Therefore, the $O_{2} Z_{2}$ and $O_{1} Z_{1}$ are different by $\widehat{\beta}_{O_{1} O_{2}}$ as shown in Fig. 1 Then, the process of transformation the Laser Scanner 2 data $\left(x_{2}^{t}(i), y_{2}^{t}(i), z_{2}^{t}(i)\right)$ into the Smart Shoe body coordinate system $\left(x_{2 S S}^{t}(i), y_{2 S S}^{t}(i), z_{2 S S}^{t}(i)\right)$ is obtained as followed:

$$
\begin{gathered}
x_{2 S S}^{t}(i)=\left(x_{2}^{t}(i)+d_{O_{1} O_{2}}\right) * \cos \left(\widehat{\beta}_{O_{1} O_{2}}\right) \\
y_{2 S S}^{t}(i)=y_{2}^{t}(i) \\
z_{2 S S}^{t}(i)=\left(x_{2}^{t}(i)+d_{O_{1} O_{2}}\right) * \sin \left(\widehat{\beta}_{O_{1} O_{2}}\right)
\end{gathered}
$$

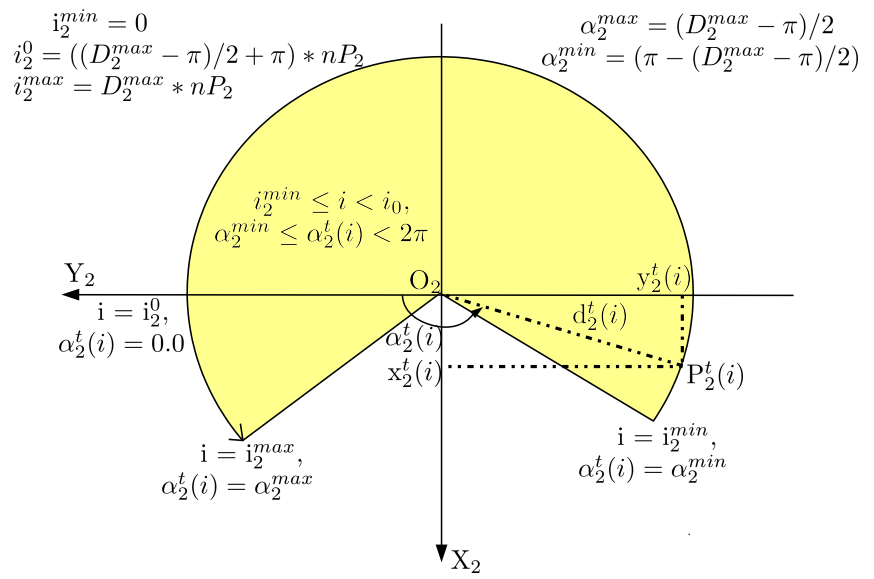

Detection Area: $0.0 \leq d A_{2}^{0} \leq 2 \pi$ Max Distance: $D_{2}^{\min } \leq d_{2}^{t}(i) \leq D_{2}^{\max } m m$

Figure 7: Field of View of the rear Laser Scanner 2.

\subsection{Transforming Laser Scanning Data into the NED frame}

This is an important step in laser scanning data processing. As discussed in the previous section, since the Smart Shoe has multiple different coordinate systems from Laser Scanner 1 frame, Laser Scanner 2 frame and IMU frame, the data collected at each frame has its own coordinates. Therefore to enable 3D mapping, we need to convert/transform them into a common frame, namely $N E D$.

It is supposed that an arbitrary point $\boldsymbol{P}_{S S}^{t}(i)=$ $\left(x_{S S}^{t}(i), y_{S S}^{t}(i), z_{S S}^{t}(i)\right)$ in the Smart Shoe body frame is any point $i$ in the data set collected from Laser Scanner 1 or Laser Scanner 2 at a any time $t$. We need to transform all points in this data set into the NED system. The transformation can be obtained as followed:

$$
\boldsymbol{P}_{e S S}^{t}(i)=\boldsymbol{M}_{N E D}^{t} * \boldsymbol{P}_{S S}^{t}(i)
$$

(4c) $_{195}$ where $\boldsymbol{M}_{N E D}^{t}=\boldsymbol{M}_{N E D}^{t \mid t}$ is a transformation matrix in Equ. 17. 


\section{Real-Time Human Foot Motion Localization Algorithm for The Smart Shoe}

As briefly discussed in subsection 2.1, the Smart Shoe is equipped with an IMU to help localize the foot motion during the walk. The scanning data is then associated with the foot position to enable 3D mapping process. However, human local-240 ization using IMU is challenging problem due to IMU drift and local magnetic disturbances from external environment.

We used the Dynamic Gait Phase (DGP) algorithm, proposed in our previous work [20], to accurately detect the stance and swing phases of the foot motion. We then use the Zero Velocity Update (ZVU) algorithm [20, 10, 21, 22] to mitigate the IMU drift. The approach is based on the fact that at each stance phase (the foot on the ground), the foot velocity should be zero. during this phase, there should be errors caused by IMU drift. Hence, combining DGP and ZVU algorithms we can significantly reduce the IMU drift problem.

Another useful observation is that, during the walk the charrection. For this reason, a Heuristic Heading Reduction (HDR) algorithm [20, 23, 21] is applied to adjust the drifted yaw angle of heading direction. If the magnetic field of environment ${ }^{24}$ makes change in a heading direction, HDR would reduce the bias of this yaw angle.

IMU device usually includes three embedded sensors: Accelerometers measuring the acceleration $\boldsymbol{a}_{b}^{\boldsymbol{t}+1}$, Gyroscopes measuring the angular rate $\boldsymbol{w}_{b}^{t+1}$, and Magnetometer measuring the magnetic field of the Earth $\boldsymbol{o}_{b}^{t+1}$. The subscript $b$ in these variThe superscript $t+1$ refers to the value of these variables at discrete time $t+1$ in the IMU's time series.

Since the IMU normally outputs data of the acceleration $\boldsymbol{a}_{b}^{t+1}$, the angular rate $\boldsymbol{w}_{b}^{t+1}$, the Earth magnetic field $\boldsymbol{o}_{b}^{t+1}$, and the 230 quaternion $\boldsymbol{q}^{t+1}$, we need to derive the Euler rotation angles, Roll $\left(\alpha^{t+1}\right)$, Pitch $\left(\beta^{t+1}\right)$ and Yaw $\left(\gamma^{t+1}\right)$, from these raw data. The detail of obtaining these rotation angles can be found in [24].

Once the DGP detected a stance phase of the foot motion at time $t+1$, the ZVU and the HDR algorithms estimate the vector $\boldsymbol{m}^{t+1}$ of the actual error measurement:

$$
\boldsymbol{m}^{t+1}=\left(\delta \psi^{t+1}, \delta \boldsymbol{w}_{b}^{t+1}, \delta \boldsymbol{v}^{t+1}\right)
$$

where, the bias error of yaw angle $\delta \psi^{t+1}$ is obtained by the HDR algorithm as:

$$
\delta \psi^{t+1}=\gamma^{T_{i}}-\frac{\gamma^{T_{(i-1)}}+\gamma^{T_{(i-2)}}}{2}
$$

where, $T_{i}$ is the last discrete time of the stance phase of step $i$.

The bias error of velocity is obtained as follows:

$$
\delta v_{b}^{t+1}=v_{b}^{t+1}-v_{a}^{t+1}
$$

where, $\boldsymbol{v}_{b}^{t+1}$ is the velocity of motion in the IMU's body frame, which can be obtained as $\boldsymbol{v}_{b}^{t+1}=\int \boldsymbol{a}_{b}^{t+1} d t . \boldsymbol{v}_{a}^{t+1}$ is the actual velocity of the foot in the stance phase, or $v_{a}^{t+1}=\left[\begin{array}{lll}0 & 0 & 0\end{array}\right]^{T}$.
The bias error of the angular rate is obtained as follows:

$$
\delta \boldsymbol{w}_{b}^{t+1}=\boldsymbol{w}_{b}^{t+1}-\boldsymbol{w}_{a}^{t+1}
$$

where, $\boldsymbol{w}_{a}^{t+1}$ is the actual angular rate of the foot in the stance phase, or $\boldsymbol{w}_{a}^{t+1}=\left[\begin{array}{lll}0 & 0 & 0\end{array}\right]^{T}$.

After applying the ZVU and the HDR algorithms to estimate the vector $\boldsymbol{m}^{t+1}$ of the actual error measurement as in Equ. 7 . we use an Extended Kalman Filter (EKF) to estimate the errors of the attitude, the angular rate, the position, the velocity, and the acceleration of the foot/shoe motion.

The EKF's error state vector at the previous time $t$ is a 15 element vector $\delta \boldsymbol{X}^{t \mid t}$, and its function corrects the INS's output values: the velocity, the position, and the attitude:

$$
\delta \boldsymbol{X}^{t \mid t}=\delta \boldsymbol{X}^{t}\left(\delta \boldsymbol{\varphi}^{t}, \delta \boldsymbol{w}^{t}, \delta \boldsymbol{p}^{t}, \delta \boldsymbol{v}^{t}, \delta \boldsymbol{a}_{b}^{t}\right)
$$

where, $\delta \boldsymbol{\varphi}^{t}, \delta \boldsymbol{w}^{t}, \delta \boldsymbol{p}^{t}, \delta \boldsymbol{v}^{t}$, and $\delta \boldsymbol{a}_{b}^{t}$ represent the EKF's estimated errors of the attitude, the angular rate, the position, the velocity, and the acceleration at the previous time $t$.

The EKF's predicted error state vector at time $t+1$ is:

$$
\delta \boldsymbol{X}^{t+1 \mid t}=\boldsymbol{\Phi}^{t+1} \delta \boldsymbol{X}^{t \mid t}+\boldsymbol{n}_{p}^{t}
$$

where, $\boldsymbol{n}_{p}^{t}$ is the process noise with a covariance matrix $\boldsymbol{Q}_{t}=$ $\boldsymbol{E}\left[\boldsymbol{n}_{p}^{t}\left(\boldsymbol{n}_{p}^{t}\right)^{T}\right]$.

The EKF's state transition matrix, $\boldsymbol{\Phi}^{t+1}$, is a $15 \times 15$ matrix:

$$
\boldsymbol{\Phi}^{t+1}=\left[\begin{array}{cccccc}
\boldsymbol{I} & \Delta t \cdot \boldsymbol{M}_{N E D}^{t+1 \mid t} & \mathbf{0} & \mathbf{0} & \mathbf{0} \\
\mathbf{0} & \boldsymbol{I} & \mathbf{0} & \mathbf{0} & \mathbf{0} \\
\mathbf{0} & \mathbf{0} & \boldsymbol{I} & \Delta t \cdot \boldsymbol{I} & \frac{-\Delta t^{2}}{2} \cdot \boldsymbol{S}\left(\boldsymbol{a}_{n^{\prime}}^{t+1}\right) \\
-\Delta t \cdot \boldsymbol{S}\left(\boldsymbol{a}_{n^{\prime}}^{t+1}\right) & \mathbf{0} & \mathbf{0} & \boldsymbol{I} & \Delta t \cdot \boldsymbol{M}_{N E D}^{t+1 \mid t} \\
\mathbf{0} & \mathbf{0} & \mathbf{0} & \mathbf{0} & -\Delta t \cdot \boldsymbol{S}\left(\boldsymbol{a}_{n^{\prime}}^{t+1}\right)
\end{array}\right]
$$

where, $\boldsymbol{I}$ is a $3 \times 3$ unit matrix, $\mathbf{0}$ is a $3 \times 3$ zero matrix. $\boldsymbol{S}\left(\boldsymbol{a}_{n^{\prime}}^{t+1}\right)$ is the skew-symmetric matrix of the accelerations:

$$
\boldsymbol{S}\left(\boldsymbol{a}_{n^{\prime}}^{t+1}\right)=\left[\begin{array}{ccc}
0 & -\boldsymbol{a}_{n^{\prime}}^{t+1}(2) & \boldsymbol{a}_{n^{\prime}}^{t+1}(1) \\
\boldsymbol{a}_{n^{\prime}}^{t+1}(2) & 0 & -\boldsymbol{a}_{n^{\prime}}^{+t+1}(2) \\
-\boldsymbol{a}_{n^{\prime}}^{t+1}(1) & \boldsymbol{a}_{n^{\prime}}^{t+1}(0) & 0
\end{array}\right]
$$

where, $\boldsymbol{a}_{n^{\prime}}^{t+1}$ is the bias-corrected acceleration in the NED frame:

$$
\boldsymbol{a}_{n^{\prime}}^{t+1}=\boldsymbol{M}_{N E D}^{t+1 \mid t} \cdot \boldsymbol{a}_{b^{\prime}}^{t+1}
$$

where, $\boldsymbol{M}_{N E D}^{t+1 \mid t}$ is the transformation matrix as in Equ. 17 .

The bias compensations for the acceleration $\boldsymbol{a}_{b^{\prime}}^{t+1}$ and angular rate $\boldsymbol{w}_{b^{\prime}}^{t+1}$ from the EKF error state vector $\delta \boldsymbol{X}^{t \mid t}$ in Equ. (11) are obtained as follows:

$$
\begin{aligned}
& \boldsymbol{w}_{b^{\prime}}^{t+1}=\boldsymbol{w}_{b}^{t+1}-\delta \boldsymbol{w}_{b}^{t+1} \\
& \boldsymbol{a}_{b^{\prime}}^{t+1}=\boldsymbol{a}_{b}^{t+1}-\delta \boldsymbol{a}_{b}^{t+1}
\end{aligned}
$$

where, the raw data of the acceleration $\boldsymbol{a}_{b}^{t+1}$, and the angular rate $\boldsymbol{w}_{b}^{t+1}$ here is in the IMU's body frame.

The transformation matrix [22, 21], $\boldsymbol{M}_{N E D}^{t+1 \mid t}$ that transforms the data from the $I M U$ 's body frame into the $N E D$ frame is obtained as follows:

$$
\boldsymbol{M}_{N E D}^{t+1 \mid t}=\boldsymbol{M}_{N E D}^{t \mid t} \cdot \frac{2 \boldsymbol{I}_{3 \times 3}+\delta \boldsymbol{\Omega}_{t} \cdot \Delta t}{2 \boldsymbol{I}_{3 \times 3}-\delta \boldsymbol{\Omega}_{t} \cdot \Delta t},
$$


where, $\delta \boldsymbol{\Omega}_{t}$ is a skew symmetric matrix of the angular rate:

$$
\delta \boldsymbol{\Omega}_{t}=\left[\begin{array}{ccc}
\mathbf{0} & -\boldsymbol{w}_{b^{\prime}}^{t}(2) & \boldsymbol{w}_{b^{\prime}}^{t}(1) \\
\boldsymbol{w}_{b^{\prime}}^{t}(2) & \mathbf{0} & -\boldsymbol{w}_{b^{\prime}}^{t}(0) \\
-\boldsymbol{w}_{b^{\prime}}^{t}(1) & \boldsymbol{w}_{b^{\prime}}^{t}(0) & \mathbf{0}
\end{array}\right]
$$

where, $\boldsymbol{w}_{b^{\prime}}^{t}$ is computed by Equ. 16 a), and $\boldsymbol{M}_{N E D}^{t \mid t}$ is the last rotation matrix updated by the $\mathrm{EKF}$ at the previous step $t$. At the first time, $t+1=1, \boldsymbol{M}_{N E D}^{1 \mid 0}$ is estimated as follows:

$$
\boldsymbol{M}_{N E D}^{1 \mid 0}=\left[\begin{array}{ccc}
c(\gamma) c(\beta) & c(\gamma) s(\alpha) s(\beta)-c(\alpha) s(\gamma) & s(\alpha) s(\gamma)+c(\alpha) c(\gamma) s(\beta) \\
c(\beta) s(\gamma) & c(\alpha) c(\gamma)+s(\alpha) s(\gamma) s(\beta) & c(\alpha) s(\gamma) s(\beta)-c(\gamma) s(\alpha) \\
-s(\beta) & c(\beta) s(\alpha) & c(\alpha) c(\beta)
\end{array}\right]
$$

here $c, s, \alpha, \beta$ and $\gamma$ are cosine (), sine (), Roll $\left(\alpha^{1}\right)$, Pitch $\left(\beta^{1}\right)_{255}$ and $\operatorname{Yaw}\left(\gamma^{1}\right)$, respectively.

The EKF's error state at time $t+1$ can be obtained as:

$$
\delta \boldsymbol{X}^{t+1 \mid t+1}=\delta \boldsymbol{X}^{t+1 \mid t}+\boldsymbol{K}^{t+1}\left[\boldsymbol{m}^{t+1}-\boldsymbol{H} \delta \boldsymbol{X}^{t+1 \mid t}\right]
$$

where, $\boldsymbol{K}_{t}$ is the Kalman gain defined in Equ. $23 ; \boldsymbol{m}^{t+1}$ is defined in Equ. (7) and $\boldsymbol{H}$ is a measurement matrix:

$$
\boldsymbol{H}_{7 \times 15}=\left[\begin{array}{lllll}
\boldsymbol{O}_{1 \times 3}^{1} & \boldsymbol{O}_{1 \times 3}^{0} & \boldsymbol{O}_{1 \times 3}^{0} & \boldsymbol{O}_{1 \times 3}^{0} & \boldsymbol{O}_{1 \times 3}^{0} \\
\boldsymbol{O}_{3 \times 3} & \boldsymbol{I}_{3 \times 3} & \boldsymbol{O}_{3 \times 3} & \boldsymbol{O}_{3 \times 3} & \boldsymbol{O}_{\boldsymbol{3}} \times 3 \\
\boldsymbol{O}_{3 \times 3} & \boldsymbol{O}_{3 \times 3} & \boldsymbol{O}_{3 \times 3} & \boldsymbol{I}_{3 \times 3} & \boldsymbol{O}_{3 \times 3}
\end{array}\right]
$$

where, $\boldsymbol{O}_{1 \times 3}^{1}=\left[\begin{array}{lll}0 & 0 & 1\end{array}\right], \boldsymbol{O}_{1 \times 3}^{0}=\left[\begin{array}{lll}0 & 0 & 0\end{array}\right], \boldsymbol{I}_{3 \times 3}$ is a $3 \times 3$ unit matrix, and $\boldsymbol{O}_{3 \times 3}$ is a $3 \times 3$ zero matrix.

The EKF's measurement model is defined by:

$$
z^{t+1}=\boldsymbol{H} \delta \boldsymbol{X}^{t+1 \mid t+1}+\boldsymbol{n}_{z}^{t+1}
$$

where $\boldsymbol{n}_{z}^{t+1}$ is the measurement noise with covariance matrix $\boldsymbol{R}^{t+1}=\boldsymbol{E}\left[\boldsymbol{n}_{z}^{t+1}\left(\boldsymbol{n}_{z}^{t+1}\right)^{T}\right]$.

The Kalman gain is obtained as follows:

$$
\boldsymbol{K}^{t+1}=\boldsymbol{P}^{t+1 \mid t} \boldsymbol{H}^{T}\left(\boldsymbol{H} \boldsymbol{P}^{t+1 \mid t} \boldsymbol{H}^{T}+\boldsymbol{R}^{t+1}\right)^{-1},
$$

where, $\boldsymbol{P}^{t+1 \mid t}$ is the estimated error covariance matrix which is computed at time $t+1$ of the IMU's output sequence:

$$
\boldsymbol{P}^{t+1 \mid t}=\Phi^{t} \boldsymbol{P}^{t \mid t}\left(\Phi^{t}\right)^{T}+\boldsymbol{Q}^{t}
$$

where, where $Q^{t}$ is the process noise covariance matrix; and the previous $\boldsymbol{P}^{t \mid t}$ is computed by:

$$
\boldsymbol{P}^{t \mid t}=\left(\boldsymbol{I}_{15 \times 15}-\boldsymbol{K}^{t} \boldsymbol{H}\right) \boldsymbol{P}^{t \mid t-1}\left(\boldsymbol{I}_{15 \times 15}-\boldsymbol{K}^{t} \boldsymbol{H}\right)^{T}+\boldsymbol{K}^{t} \boldsymbol{R}^{t}\left(\boldsymbol{K}^{t}\right)^{T}
$$

The acceleration $\boldsymbol{a}_{e}^{t+1}$ of human motion in the NED frame can be obtained by transforming the bias-compensated acceleration from Equ. (16) to the $N E D$ frame then subtracting the vector $\boldsymbol{g}_{e}$ from it as follows:

$$
\boldsymbol{a}_{e}^{t+1}=\boldsymbol{M}_{N E D}^{t+1 \mid t} \cdot \boldsymbol{a}_{b^{\prime}}^{t+1}-\boldsymbol{g}_{e} .
$$

The predicted velocity of the EKF in the $N E D$ frame at time $t+1$ is integrated from an motion acceleration as:

$$
\boldsymbol{v}^{t+1 \mid t}=\boldsymbol{v}^{t \mid t}+\boldsymbol{a}_{e}^{t+1} \cdot \Delta t .
$$

This velocity is integrated one more time to compute the foot position in the $N E D$ frame:

$$
p^{t+1 \mid t}=p^{t \mid t}+v^{t+1 \mid t} \cdot \Delta t
$$

Finally, we can use the EKF's error state vector in Equ. (20) to adjust the values of the velocity in Equ. (27), the position in Equ. (28), and the attitude in Equ. (17):

$$
\begin{gathered}
\boldsymbol{v}^{t+1 \mid t+1}=\boldsymbol{v}^{t+1 \mid t}-\delta \boldsymbol{v}^{t+1 \mid t+1} \\
\boldsymbol{p}^{t+1 \mid t+1}=\boldsymbol{p}^{t+1 \mid t}-\delta \boldsymbol{p}^{t+1 \mid t+1}
\end{gathered}
$$

\section{Real-time 3D Mapping Algorithm}

\subsection{Integrating Laser Data and Foot Motion Trajectory}

Laser data point $\boldsymbol{P}_{e S S}^{t}(i)$ in the NED system needs to be integrated with the foot motion trajectory to build a correct map of environments. Because the Smart Shoe body frame centered at $O_{S}$ is equivalent to the $I M U$ body frame centered at $O_{I}$, the trajectory of foot motion based on the movement of Smart Shoe rigid body system $O_{S}$ is equivalent to $O_{I}$. This also means that the real-time position of $O_{I}$ or trajectory of foot movement is the root for every laser point $\boldsymbol{P}_{e S S}^{t}(i)$ in a 3D map. Hence, we can compute the real-time position of all 3D mapping points of the surrounding environment along with the foot movement $\boldsymbol{P}_{N E D 3 D}^{t}(i)$ :

$$
\boldsymbol{P}_{N E D 3 D}^{t}(i)=\boldsymbol{P}_{e S S}^{t}(i)+\boldsymbol{p}^{t}
$$

where, the $\boldsymbol{p}^{t}=\boldsymbol{p}^{t \mid t}$ is a 3D position on the human foot motion trajectory at time $t$ in the $N E D$ frame.

\subsection{Real-time 3D Mapping Algorithm}

The real-time 3D mapping algorithm, which is designed in Robotic Operating System (ROS) environment, is presented in Fig. 8. The algorithm starts at the Start event to mount Laser Scanner 1, 2 and IMU sensor. The result of this event is three new Listener nodes: $L_{2}$-Listener, $L_{1}$-Listener and I-Listener for Laser Scanner 1, 2 and IMU, respectively. Each of them operates independently and parallel to each other.

Because their output data rate is different, the waiting time $\delta_{1}^{t}, \delta_{2}^{t}$, and $\delta_{I}^{t}$ are different. Whenever these devices finish their data collection phase, they send an interruption to their Listener nodes. Then these agents describe the collected data to other modules waiting to process this data.

In this diagram (Fig. 8, Comp $\boldsymbol{P}_{2}^{t}(i), \operatorname{Comp} \boldsymbol{P}_{1}^{t}(i)$ and EKF INS $\boldsymbol{p}^{t}$ are the processing modules. The modules $\boldsymbol{P}_{1}^{t}(i)$ and $\boldsymbol{P}_{2}^{t}(i)$ process the stream data from Laser Scanner 1 and 2 , respectively. They convert the distant point $i$ in the scanning data at time $t$ into the 3D coordinate data in the Smart Shoe body frame by the Equ. (2), (3), (4), and (5). Then, the module $\boldsymbol{P}_{e S S}^{t}(i)$ transforms this data from the Smart Shoe frame into the NED system by Equ. (6).

The I - Listener node of the IMU sensor gets the stream data from the IMU and then describes it to EKF INS $\boldsymbol{p}^{t}$ module. This module uses the acceleration, angle rate, and magnetic data from the IMU description to output the velocity and position of the foot motion. This process is supported by the EKF filter presented in the previous section. The EKF INS filter helps minimize accumulated errors from IMU drift over time. 


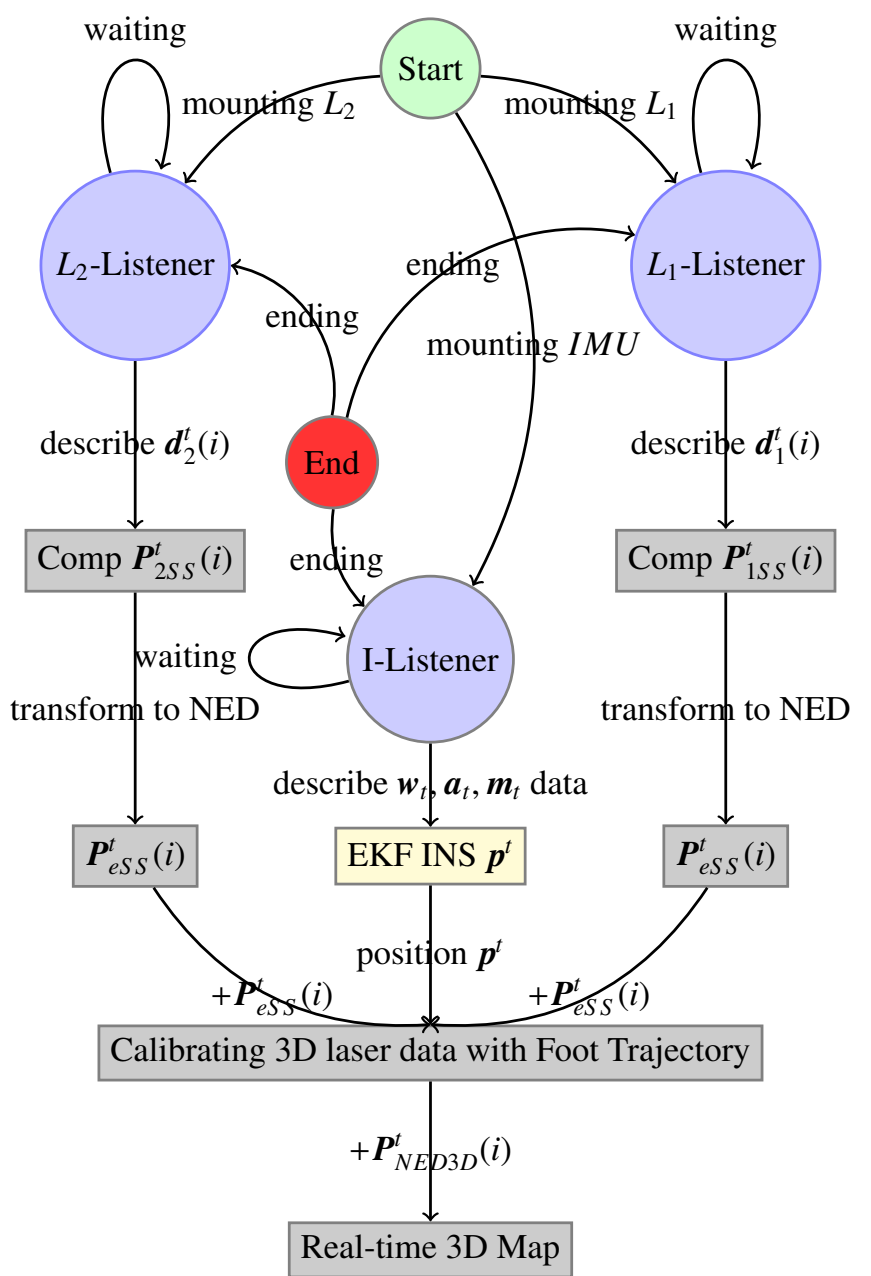

Figure 8: A Real-time 3D Mapping Algorithm.

This EKF INS $\boldsymbol{p}^{t}$ module outputs an accurate trajectory data of foot motion.

The module "Calibrating 3D laser data with Foot Trajectory" processes the calibration of the foot trajectory data with 3D laser data to build a 3D map of the environment.

\section{Experimental Results}

We implemented the Smart Shoe on a Puma sport shoe with a white soft ring around its header as shown in Fig. 9 On the white soft ring, we attached two Hokuyo URG-04LX-UG01 laser scanners and one MicroStrain 3DM-GX3-25 IMU sensor.

The design and implementation of the white soft ring follows all requirements of the Smart Shoe design in Fig. 1 Namely, Hokuyo 1 is attached at the Laser Scanner 1 position, and Hokuyo 2 is attached at the Laser Scanner 2 position, and MicroStrain 3DM-GX3-25 IMU is attached under the Hokuyo 1.

The white soft ring in this implementation is tied onto the shoe by the shoe string. This white soft ring has two roles. It is hard enough to be able to attach all devices on the shoe tightly.

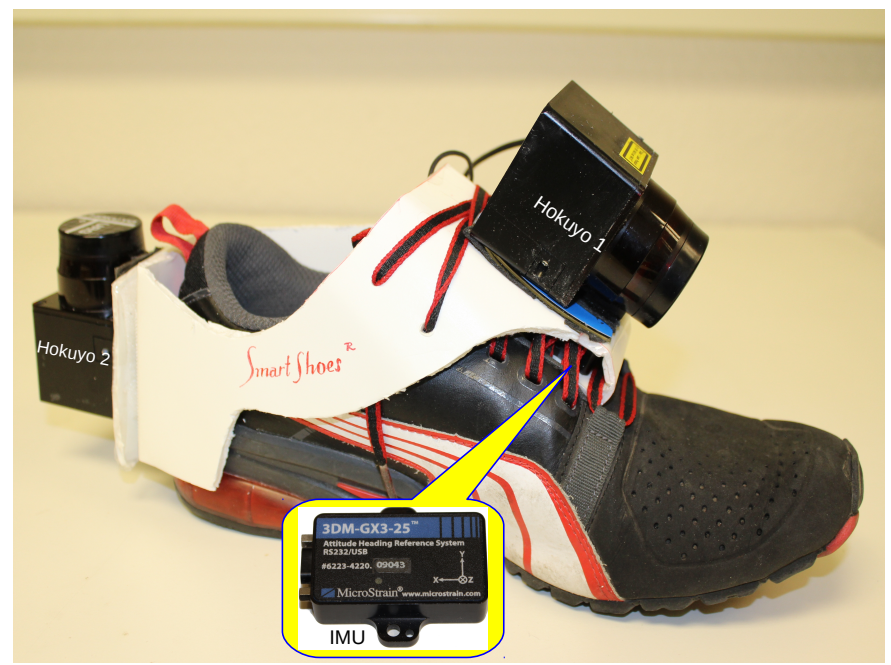

Figure 9: A Smart Shoe implementation.

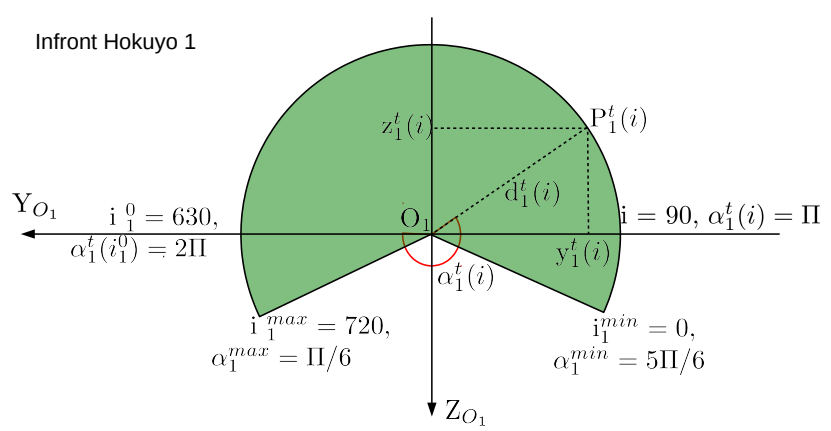

$\mathrm{x}_{1}^{t}(i)=0.0$

$y_{1}^{t}(i)=d_{1}^{t}(i) * \cos \left(\alpha_{1}^{t}(i)\right)$

$z_{1}^{t}(i)=d_{1}^{t}(i) * \sin \left(\alpha_{1}^{t}(i)\right)$

$$
\begin{aligned}
& \text { Where } \mathrm{i} \quad \text { and } \alpha_{1}^{t}(i): \\
& \quad \mathrm{i}_{1}^{\min }<=i<=i_{1}^{0}, 5 \Pi / 6<=\alpha_{1}^{t}(i)<=2 \Pi \\
& i_{1}^{0}<=i<=i_{1}^{\max }, 0<=\alpha_{1}^{t}(i)<=\Pi / 6 \\
& 0.0<=d_{1}^{t}(i)<=4,000 \mathrm{~mm}
\end{aligned}
$$

Figure 10: Converting scanning points into 3D body frame of Hokuyo 1.

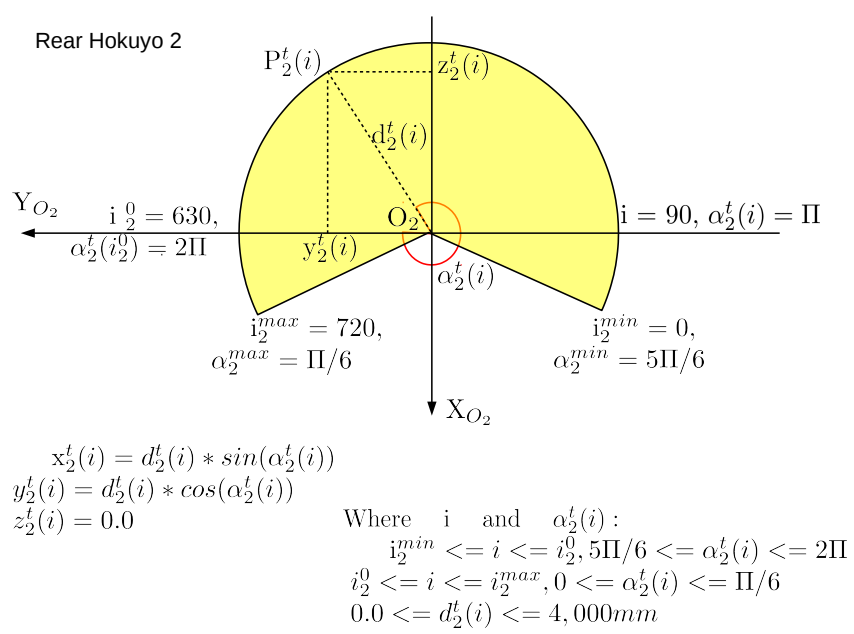

Figure 11: Converting scanning points into 3D body frame of Hokuyo 2. 

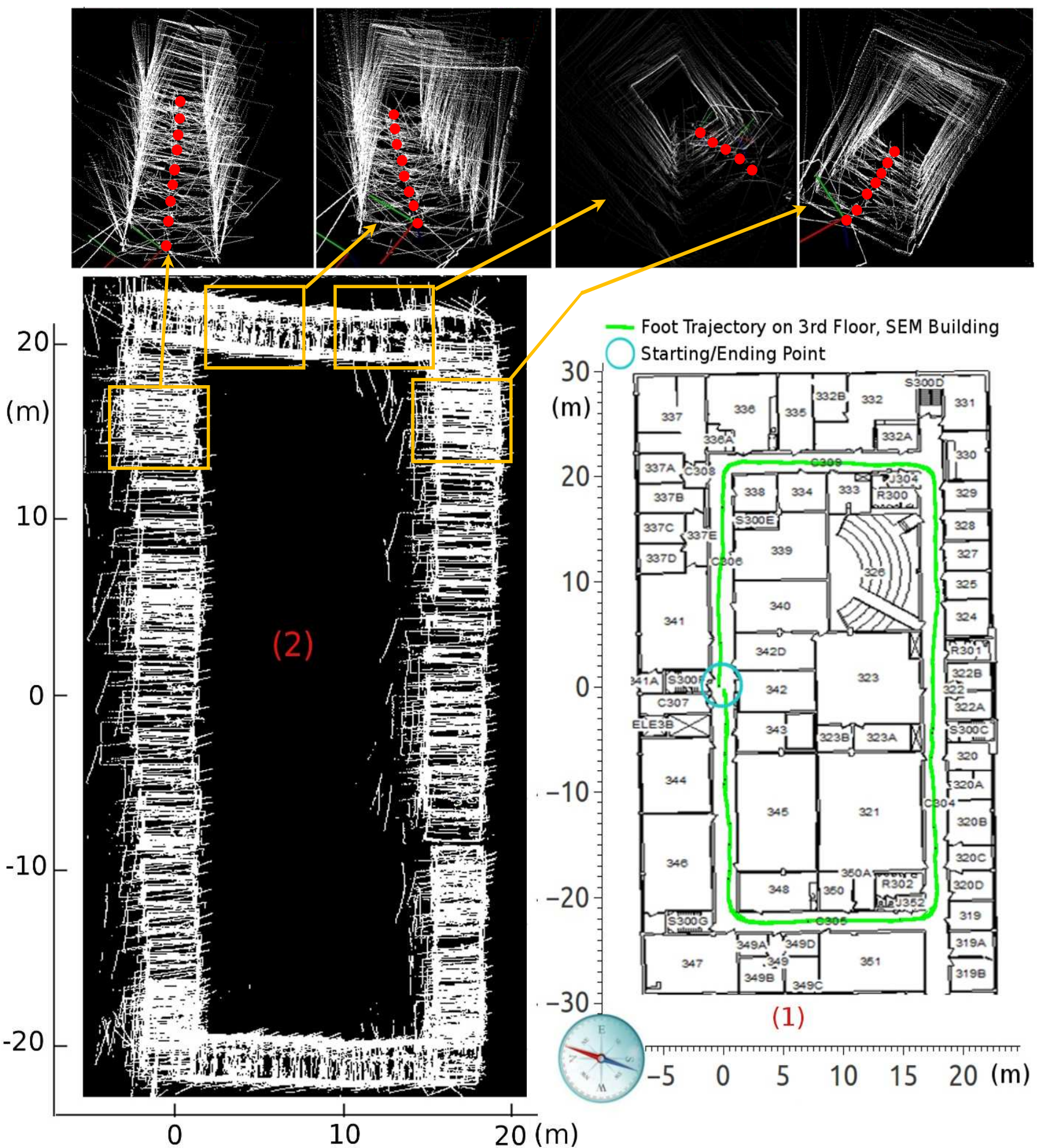
Starting/Ending Point
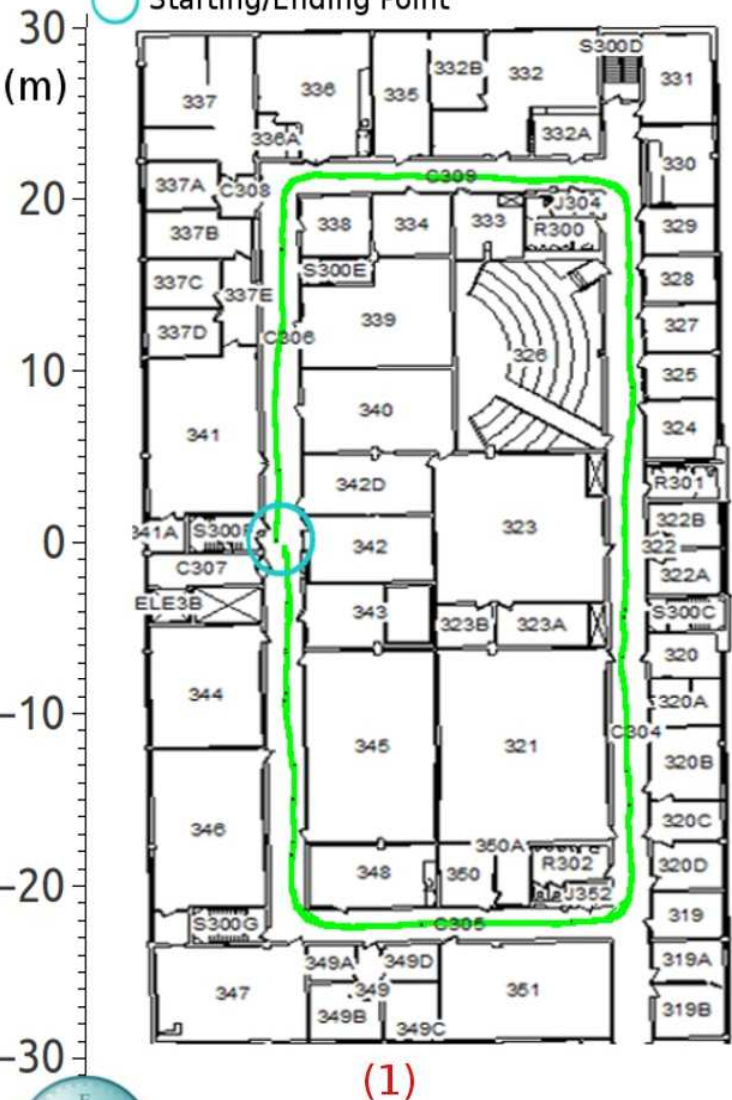

\section{(1)}

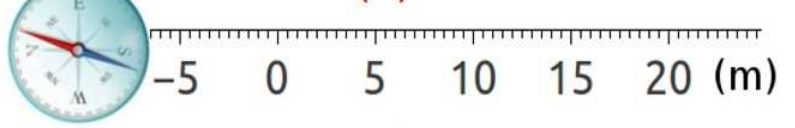

Figure 12: The obtained 3D map of the 3rd floor hallway associated with foot's trajectory in the SEM building by using our Smart Shoe. (1) Trajectory of the foot during walking in a closed loop with an indication of starting and ending positions. (2) A 3D map of the hallway during walking with some zoom-in areas.

Hokuyo 2 body frame and the Smart Shoe body frame. The white soft ring here also makes the Smart Shoe light and comfortable for wearing, and it can be removed from the shoe easily. 315 All integrated devices can be connected to a computer through wireless connections such as BLE or NFC for mobility. However, for simplicity, we used USB connection among them.

Two Hokuyo laser scanners in this implementation have the identical technical specifications. The maximum scanning range $D_{1}^{\max }, D_{2}^{\max }$ is $240^{\circ}$. The number of scanning points $n P_{1}, n P_{2}$ for each degree of scanning detection area is approximated 3 points. The maximum detection distance $d_{1}^{t}(i), d_{2}^{t}(i)$ is less than or equal to $4,000 \mathrm{~mm}$. Time of one full $240^{\circ} \mathrm{scan}$ is nearly $0.8 \mathrm{~s}$. The distance $d_{\mathrm{O}_{1} \mathrm{O}_{2}}$ is $0.22 \mathrm{~m}$. The different angle $\widehat{\beta}_{O_{1} O_{2}}$ is $\pi / 6$. 
We show the technical specification and implementation of these Hokuyo laser scanners and how to calculate 3D positions of any point $i$ from any scanning time $t$ in Fig. 10 and Fig. 11. 360

All collected data is processed by the proposed real-time mapping algorithm as presented in Fig. 8. This algorithm is 325 coded in $\mathrm{C}++$ on the ROS platform. We conducted the experiment of the Smart Shoe inside the $3^{\text {rd }}$ floor in the Scrugham Engineering and Mines (SEM) building, University of Nevada, Reno (UNR) campus. The Smart Shoe collected more than 3 million 3D points for this hallway and the size of the raw data set for one time collection is over $135 \mathrm{Mb}$.

Fig. 12 shows the obtained result of a 3D map of the SEM hallway along during walking. The user is walking in a closed $d_{370}$ loop along the hallway, and his walking trajectory is plotted in Fig. 12(1). As can be seen, the starting and ending positions are close to each other, and the walking trajectory matches well with the floor plan. This clearly shows the high accuracy of the foot localization. During walking, the Smart Shoe collects laser scanning data and builds a 3D map in real-time as shown in Fig. 12 (2). To intuitively demonstrate the quality of the 3D map, some zoom-in areas of the map are presented at the top of this figure.

To better illustrate the $3 \mathrm{D}$ mapping result, we show both $3 \mathrm{D}_{380}$ map and visual image of the hallway as presented in Fig. 13 We can see that the structure of the built 3D map is similar to the visual image. Since this is an intuitive comparison, in the future work we will develop a ground truth model using terrestrial scanning (i.e. based on ) to evaluate accuracy of the ${ }_{385}$ mapping results from our Smart Shoe.

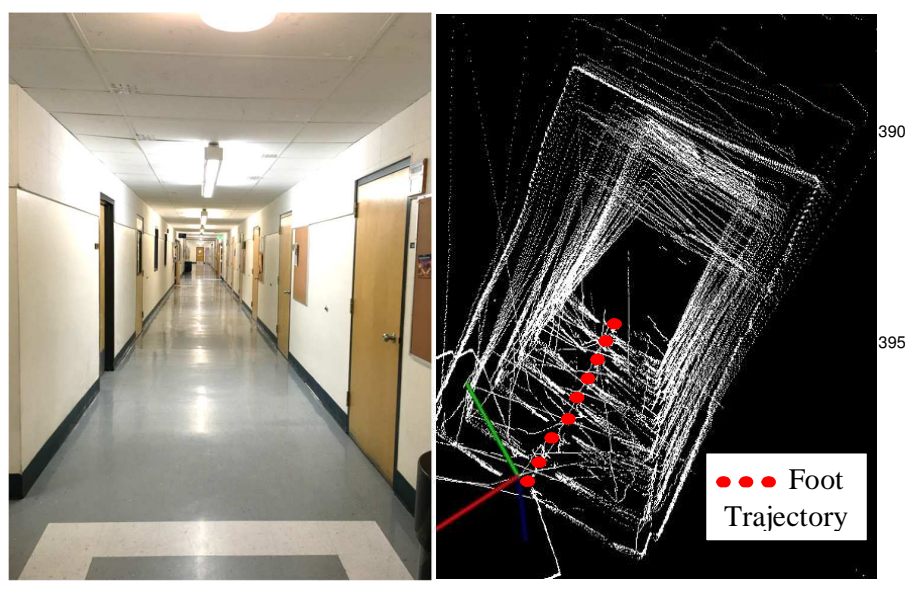

Figure 13: Visual image of the hallway versus the 3D map.

400

\section{Conclusion and Discussion}

This paper proposed a new comfortable wearing device, Smart Shoe, for building a real-time 3D map of the surrounding ${ }^{405}$ environment. By wearing this shoe, the user can work normally and build a real-time 3D map. Overall design of the Smart Shoe with details of sensor integration, data collection, transformation and processing was reported. To enable $3 \mathrm{D}$ scanning data ${ }^{410}$ registration, a foot localization algorithm was proposed and integrated to accurately track and localize the shoe/foot motion.
A 3D real-time mapping algorithm was introduced to build a map of environments. Experimental results of the foot motion localization and 3D mapping are conducted to demonstrate the proposed Smart Shoe.

The success of this Smart Shoe could open up a wide range of possible applications.

In the Architecture, Engineering, and Construction (AEC) domain, the Smart Shoe can be used for floor plan surveying, in-construction monitoring, planning renovations, space usage planning, managing building maintenance, just to mention a few. The low cost, lightweight, and unobtrusive virtues of the Smart Shoe make it a suitable site surveillance tool in construction fields. The shoes, for example, can serve as a platform for building and collecting 3D models of small constructions sites, from which a large-scale construction field model can be constructed cost-effectively, efficiently, accurately and timely. Such an application can be realized by equipping a number of construction workers with the Smart Shoe.

In addition, the real-time outputs from the Smart Shoe can also be used for construction progress monitoring, from which construction planning and management can benefit accordingly. The depiction of the state of the facility as it was actually built is valuable information for construction manager. For instance, once the foundation worker's shoes report that the foundation is at $90 \%$ of completion, the construction manager can initiate the foundation inspection process so that it can be started right after the foundation is built, shortening the waiting period. The shoes' real-time output can also be used for clash detection, which is the collision between built components and those in a design model. The key advantage here is the ability to detect such collision in timely manner, during the construction, so that correction can be done early on, cutting down the cost and reduce the possibility of unexpected delays during construction.

Another application of our proposed device is on construction safety monitoring and training. Since the shoes can indicate both the motion of the workers and their surrounding, its output can be used to tell when the worker is following specific safety and health code. If a series of non-safe practice observed by the shoes, it can send real-time report to the worker's supervisors or colleagues to prevent incidents. In essence, a closedloop feedback algorithm can be developed based on the Smart Shoe for detecting and preventing unsafe practices in real-time.

\section{References}

[1] Automatic creation of semantically rich $3 \mathrm{~d}$ building models from laser scanner data, Automation in Construction 31 (2013) 325 - 337.

[2] Mobile 3d mapping for surveying earthwork projects using an unmanned aerial vehicle (uav) system, Automation in Construction 41 (2014) 1 - 14.

[3] Automatic reconstruction of as-built building information models from laser-scanned point clouds: A review of related techniques, Automation in Construction 19 (7) (2010) 829 - 843.

[4] Velodyne, Velodyne lidar available, http://www.velodynelidar.com/lidar/lidar.aspx (2014) 1.

[5] Z. Chong, B. Qin, T. Bandyopadhyay, M. Ang, E. Frazzoli, D. Rus, Synthetic $2 \mathrm{~d}$ lidar for precise vehicle localization in $3 \mathrm{~d}$ urban environment, 2013 IEEE International Conference on Robotics and Automation (ICRA) (2013) 1554-1559. 
[10] I. Skog, P. Handel, J. Nilsson, J. Rantakokko, Zero-velocity detection algorithm evaluation, Biomedical Engineering, IEEE Transactions 57 (11) (2010) 2657-2666.

[11] G. Cai, B. M. Chen, T. H. Lee, Unmanned rotorcraft systems, Advances in Industrial Control, Springer-Verlag London Limited (2011) 2657-2666.

[12] S. Song, H. Geyer, Regulating speed and generating large speed transitions in a neuromuscular human walking model, IEEE International Conference on Robotics and Automation 511-516.

[13] M. P. Murray, L. A. Mollinger, G. M. Gardner, S. B. Sepic, Kinematic and ${ }^{500}$ emg patterns during slow free and fast walking, Orthopaedic Research 2 (1984) 272-280.

[14] L. V. Nguyen, H. M. La, Development of a smart shoe for building a real-time $3 \mathrm{~d}$ map, in Proceedings of The 32st International Symposium on Automation and Robotics in Construction and Mining (ISARC), Oulu, Finland (2015) 1-8

[15] P. Fritzson, Principles of Object-Oriented Modeling and Simulation with Modelica 2.1, IEEE Press, US, 2004.

[16] H. Zhang, J. Qian, L. Shen, Y. Zhang, Research on healthy subject gait cycle phase at different walking speeds, Robotics and Biomimetics (ROBIO), 2012 IEEE International Conference on (2012) 1349-1354.

[17] R. Zhang, C. Vogler, D. Metaxas, Human gait recognition, Computer Vision and Pattern Recognition Workshop, 2004. CVPRW '04. Conference on (2004) 18-18.

[18] W. George, I. Collins, The Foundations of Celestial Mechanics, The Pachart Foundation dba Pachart Publishing House and reprinted by permission, US, 2004.

[19] R. S. Lim, H. M. La, W. Sheng, A robotic crack inspection and mapping system for bridge deck maintenance, Automation Science and Engineering, IEEE Transactions 11 (2) (2014) 367-378.

455 [20] L. V. Nguyen, H. M. La, T. H. Duong, Dynamic human gait phase detection algorithm, The ISSAT International Conference on Modeling of Complex Systems and Environments (MCSE), Danag, Vietnam (2015)520 $1-8$.

[21] A.R.Jimenez, F. Seco, J. Prieto, J.Guevara, Indoor pedestrian navigation using an ins/ekf framework for yaw drift reduction and a foot-mounted imu, IEEE Positioning Navigation and Communication (WPNC), 7th Workshop 135-143.

[22] E. Foxlin, Pedestrian tracking with shoe-mounted inertial sensors, Com- ${ }^{525}$ puter Graphics and Applications, IEEE 25 38-46.

[23] J. Borestein, L. Ojeda, S. Kwanmuang, Heuristic reduction of gyro drift in imu-based personnel tracking system, SPIE Defense, Security and Sensing Conference 7306 (2009) 1-11.

[24] L. V. Nguyen, H. M. La, Real-time human foot motion localization algorithm with dynamic speed, IEEE Transactions on Human-Machine Sys- ${ }^{530}$ tems (Accepted) (2016) 1-12.

Luan V. Nguyen received the B.S. and M.S. in Computer Science from Vietnam National University, Hanoi, Vietnam in 535 1999 and 2006, respectively. He earned his M.S. in Computer Science from Rutgers University, New Jersey, USA, in 2014. He is now working toward his PhD study at the Advanced Robotics and Automation (ARA) 54 Lab, Department of Computer Science and Engineering, University of Nevada, Reno, NV, USA. His research interests include human-machine systems, robotics, and human-robot interactions.
Hung M. La received the B.S. and M.S. degrees in Electrical Engineering from Thai Nguyen University of Technology, Thai Nguyen, Vietnam, in 2001 and 2003, respectively, and the Ph.D. degree in Electrical and Computer Engineering from Oklahoma State University, Stillwater, OK, USA, in 2011.

$\mathrm{He}$ is an Assistant Professor and Director of the Advanced Robotics and Automation (ARA) Lab at the Department of Computer Science and Engineering, University of Nevada, Reno, NV, USA. From 2011 to 2014, he was a Post Doctor and then a Research Faculty at the Center for Advanced Infrastructure and Transportation (CAIT), Rutgers University, New Jersey, USA. He has been actively involved in research projects with the U.S. Department of Transportation (DoT), Department of Defense (DoD), and National Science Foundation (NSF). He has authored over 50 papers published in major journals, book chapters and international conference proceedings. His current research interests include robotic systems, mobile sensor networks, and intelligent transportation systems.

Dr. La is the recipient of the 2014 American Society of Civil Engineer's Charles Pankow Award for the Robotics Assisted Bridge Inspection Tool (RABIT), and three best conference paper awards. Dr. La is an Associate Editor of the IEEE Transactions on HumanMachine Systems; and Guest Editor of the International Journal of Robust and Nonlinear Control; and an Editorial Board member of the International Journal of Automation and Control, and the International Journal of Robotic Engineering.

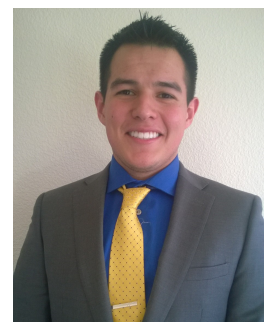

Jesus Sanchez received the B.S. degree in Computer Science and Engineering from University of Nevada, Reno, NV, USA, in December 2015. He is currently a robotic software developer at Bastian Solutions, Inc., Dallas, Texas, USA. He joined the ARA Lab, Department of Computer Science and Engineering, University of Nevada, Reno as an undergraduate student in January 2015. His research interests include robotics, computer vision and artificial intelligence

Tam Vu received the B.S in Computer Sci-

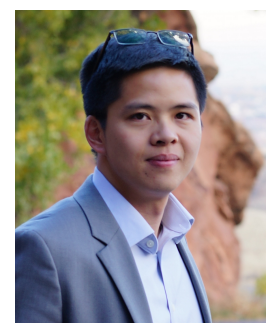
ence from Hanoi University of Technology, Vietnam in 2006, and the Ph.D. in Computer Science from WINLAB, Department of Computer Science, Rutgers University, New Jersey, USA, in 2013.

He is currently an Assistant Professor and Director of the Mobile and Networked Systems Lab at the Department of Computer Science, University of Colorado Denver since 2013. He is the recipient of CRC Interdisciplinary Fellowship at UC Denver 2015. He received Google Faculty Research Award in 2014 for his work in Chrome browser authentication. He received best paper award for inventing new form of communication, called Capacitive Touch Communication, in ACM MobiCom 2012. He was also a recipient of ACM MobiCom 2011 best paper award for his work on driver phone use detection. His research also received wide press coverage including CNN TV, NY Times, The Wall Street Journal, National Public Radio, MIT Technology Review, Yahoo News, among other venues. 